\title{
Progress towards steady-state operation and real-time control of internal transport barriers in JET
}

\author{
X. Litaudon ${ }^{1}$, A. Bécoulet ${ }^{1}$, F. Crisanti ${ }^{2}$, R.C. Wolf ${ }^{3}$, \\ Yu.F. Baranov ${ }^{4}$, E. Barbato ${ }^{2}$, M. Bécoulet ${ }^{1}$, R. Budny ${ }^{5}$, \\ C. Castaldo $^{2}$, R. Cesario ${ }^{2}$, C.D. Challis ${ }^{4}$, G.D. Conway ${ }^{6}$, \\ M.R. De Baar ${ }^{7}$, P. De Vries ${ }^{7}$, R. Dux ${ }^{6}$, L.G. Eriksson ${ }^{1}$, \\ B. Esposito ${ }^{2}$, R. Felton ${ }^{4}$, C. Fourment ${ }^{1}$, D. Frigione ${ }^{2}$, X. Garbet ${ }^{1}$, \\ R. Giannella ${ }^{1}$, C. Giroud ${ }^{7}$, G. Gorini ${ }^{8}$, N.C. Hawkes ${ }^{4}$, T. Hellsten ${ }^{9}$, \\ T.C. Hender ${ }^{4}$, P. Hennequin ${ }^{1}$, G.M.D. Hogeweij ${ }^{7}$, \\ G.T.A. Huysmans ${ }^{1}$, F. Imbeaux ${ }^{1}$, E. Joffrin ${ }^{1}$, P.J. Lomas ${ }^{4}$, \\ Ph Lotte ${ }^{1}$, P. Maget ${ }^{1}$, J. Mailloux ${ }^{4}$, P. Mantica ${ }^{8}$, M.J. Mantsinen ${ }^{10}$, \\ D. Mazon ${ }^{1}$, D. Moreau ${ }^{1}$, V. Parail ${ }^{4}$, V. Pericoli ${ }^{2}$, E. Rachlew ${ }^{9}$, \\ M. Riva ${ }^{2}$, F. Rimini ${ }^{1}$, Y. Sarazin ${ }^{1}$, B.C. Stratton ${ }^{5}$, T.J.J. Tala ${ }^{10}$, \\ G. Tresset $^{1}$, O. Tudisco ${ }^{2}$, L. Zabeo ${ }^{1}$, K.-D. Zastrow ${ }^{4}$ and \\ JET-EFDA contributors

\footnotetext{
${ }^{1}$ Association EURATOM-CEA, CEA Cadarache, F-13108 St Paul lez Durance, France

${ }^{2}$ Associazione EURATOM-ENEA sulla Fusione, Centre Ricerche Frascati, Italy

${ }^{3}$ Institut für Plasmaphysik, Association EURATOM-FZJ, D-52425 Jülich, Germany

${ }^{4}$ UKAEA-EURATOM Association, Culham Science Centre, Abingdon, OX14 3DB, UK

${ }^{5}$ Princeton Plasma Physics Laboratory, PO Box 451, Princeton, NJ 08543, USA

${ }^{6}$ Max-Planck-Institut für Plasmaphysik, EURATOM-Assoziation, Garching, Germany

${ }^{7}$ Ass. EURATOM-FOM, TEC, Cluster, 3430 BE Nieuwegein, The Netherlands

${ }^{8}$ Instituto di Fisica del Plasma, EURATOM-ENEA-CNR Association, Milan, Italy

${ }^{9}$ Physics Deptartment, KTH, Association EURATOM/VR, S-10044 Stockholm, Sweden

${ }^{10}$ Association EURATOM-TEKES, VTT Chemical Technology, FIN-02044, Finland

E-mail: xavier.litaudon@cea.fr
}

Received 15 October 2002, accepted for publication 23 May 2003

Published 23 June 2003

Online at stacks.iop.org/NF/43/565

\begin{abstract}
In JET, advanced tokamak research mainly focuses on plasmas with internal transport barriers (ITBs) that are strongly influenced by the current density profile. A previously developed optimized shear regime with low magnetic shear in the plasma centre has been extended to deeply negative magnetic shear configurations. High fusion performance with wide ITBs has been obtained transiently with negative central magnetic shear configuration: $H_{\text {IPB } 98(y, 2)} \sim 1.9$, $\beta_{\mathrm{N}}=2.4$ at $I_{\mathrm{p}}=2.5 \mathrm{MA}$. At somewhat reduced performance, electron and ion ITBs have been sustained in full current drive operation with $1 \mathrm{MA}$ of bootstrap current: $H_{\mathrm{IPB} 98(\mathrm{y}, 2)} \sim 1, \beta_{\mathrm{N}}=1.7$ at $I_{\mathrm{p}}=2.0 \mathrm{MA}$. The ITBs were maintained for up to $11 \mathrm{~s}$ for the latter case. This duration, much larger than the energy confinement time (37 times larger), is already approaching a current resistive time. New real-time measurements and feedback control algorithms have been developed and implemented in JET for successfully controlling the ITB dynamics and the current density profile in the highly non-inductive current regime.
\end{abstract}

PACS numbers: 52.25.Xz, 52.55.Fa, 52.50.Sw, 52.55.Wq, 52.25.Fi, 52.35.Hr, 52.50.Gj

\section{Introduction}

Improvement of the tokamak concept in terms of confinement and stability is a crucial challenge that could lead to operating the device in a continuous mode. In a steady-state tokamak reactor, the plasma current is entirely sustained by noninductive current drive means and the self-generated bootstrap current provides a significant fraction of the plasma current. 
A major challenge remains to extend the high-performance regimes obtained in present-day tokamaks towards genuine steady-state conditions where the current density profile is non-inductively driven. The development of steady-state operational regimes with improved confinement and stability is known as 'advanced tokamak' research (e.g. [1-4]). This paper reports on the recent (2000-2002) progress achieved on JET towards controlled steady-state regimes.

In JET, advanced tokamak research mainly focuses on plasmas with internal transport barriers (ITBs) that are strongly influenced by the current density profile [5,6]. ITBs are qualitatively defined as regions in the plasma core where the radial anomalous thermal transport (and/or particle) of the conventional confinement mode (L-mode) is significantly reduced. Moreover, a quantitative definition of ITBs has been proposed and successfully applied to characterize JET ITBs (emergence, strength and space-time evolution) in a wide range of plasma parameters [7]. It was found that the dimensionless local parameter, $\rho_{\mathrm{T}}^{*}=\rho_{\mathrm{s}} / L_{\mathrm{T}}$, characterizes the ITB features with a low computational cost $\left(\rho_{\mathrm{s}} / L_{\mathrm{T}}\right.$ is the local ion Larmor radius at the speed of sound normalized to the (electron or ion) temperature gradient scale length $L_{\mathrm{T}}$ ). An ITB exists when the normalized Larmor radius, $\rho_{\mathrm{T}}^{*}$, exceeds some critical value, $\rho_{\mathrm{ITB}}^{*}$, i.e. $\rho_{\mathrm{T}}^{*} \geqslant \rho_{\mathrm{ITB}}^{*}$. A possible interpretation leading to the theoretical relevance of this dimensionless criterion as a local indicator of a bifurcated plasma state is the stabilization of turbulence by the $E \times B$ rotational flow shear. By resorting to a dimensional analysis, it is possible to recast the $E \times B$ stabilization criterion (the $E \times B$ shear exceeding a typical linear growth rate) in the form of $\rho_{\mathrm{T}}^{*} \geqslant \rho_{\mathrm{ITB}}^{*}$. Dependence of $\rho_{\text {IтB }}^{*}$ on some dimensionless parameters are, in principle, possible. In practice, a systematic and statistical analysis of 116 JET discharges has shown that the proposed criterion characterizes well the appearance of ITBs with a critical value of $\rho_{\text {ITB }}^{*}=0.014$ [7]. Once validated, this practical ITB criterion has been used routinely to speed up the identification of the experimental database. In addition, the calculation of this normalized temperature gradient has been implemented in the JET real-time system in view of characterizing the ITB features and ultimately controlling their dynamics with feedback loops.

The paper is organized as follows. In section 2, the achievement of (transient) high fusion performance with non-monotonic $q$-profiles (negative central magnetic shear configuration) is reported [8]. The influence of the $q$-profile in the plasma performance is also stressed. Section 3 describes the quasi-stationary operation with a high fraction of non-inductive current $[9,10]$. Finally, recent and major developments to control in real-time with closed feedback loops, the local ITB or current profile characteristics are reviewed in section 4 [11].

\section{High fusion performance plasmas with negative central magnetic shear}

A previously developed optimized shear regime with low or weakly negative magnetic shear in the plasma centre $[3,12,13]$ has been extended to noticeably negative central magnetic shear configurations $[8,14-17]$. The $q$-profile has been varied using mainly the LHCD during the initial current ramp-up phase of the plasma discharge, prior to the application of the high-power heating. A dedicated set of experiments has been performed to reveal the role played by the $q$-profile in the formation of ITBs and their performances [8]. The magnetic shear in the plasma interior was varied from small and positive, in the Ohmic preheat case, to weakly to highly negative ('current hole') by increasing the LHCD power and/or optimizing the plasma initiation (figure 1 (left)). In addition, the injected torque in the co-current direction has been systematically varied for each $q$-profile $\left(B_{0}=2.6 \mathrm{~T}\right.$, $I_{\mathrm{p}} \sim 2.2 \mathrm{MA}$ ). Figure 1 (right) shows the peak value of $\rho_{\mathrm{Te}}^{*}$ applied to the electron temperature profile in the region of 'wide' transport barrier $(r / a>0.5)$, as a function of additional power for the different $q$-profiles and injected torque. The wide ITBs required for high fusion performance are formed close to the low-order rational $q=2$ surface for both monotonic [18] and non-monotonic $q$-profiles [19]. The plasmas with an LHCD prelude tend to provide a 'stronger' electron ITB (e.g. larger value $\rho_{\mathrm{Te}}^{*}$ ) than the Ohmic preheat cases. This observation is attributed to the reduction of the magnetic shear at the location of the low-order rational safety factor surface (e.g. $q=2$ ), which is expected to be favourable for the formation of the ITBs and their performances [20,21]. First, the high wavenumber ITG/TEM growth rates decrease when the magnetic shear is lowered, as recently discussed for the JET discharges by Garbet et al [21]. Second, turbulence simulations also indicate that a low magnetic shear close to low-order rational $q$-surfaces favours the development of a region without any low wavenumber resonant surface [21].

Following this approach, but at a higher toroidal field $\left(B_{0}=3.45 \mathrm{~T}\right)$, high fusion performance has been achieved with negative central magnetic shear with a minimum $q$-value, $q_{\text {min }}$, approaching two (figure 2 (left)) [8]. The LH power (2-3 MW) was applied soon after plasma initiation and until the main heating phase. A narrow ITB is first visible on the electron temperature profile during the LHCD prelude phase, which persists during the early part of the main heating phase. Then, a wide ITB with a very steep gradient develops on the thermal pressure profiles, and is thought to be triggered when $q_{\min }$ reaches two. ITBs at large plasma radius contribute significantly to the enhancement of fusion performance: total neutron yield, $R_{\mathrm{NT}}=4.1 \times 10^{16}$ neutrons s ${ }^{-1} ; H_{\mathrm{ITER}-89 \mathrm{P}} \sim$ $3.3, H_{\mathrm{IPB} 98(\mathrm{y}, 2)} \sim 1.9 ; \beta_{\mathrm{N}}=2.4$ at $I_{\mathrm{p}}=2.5 \mathrm{MA}\left(q_{95}=4.5\right)$. The transient high-performance phase is completed with a large ELM followed by a disruption (global pressure $n=1$ kink mode instability). A further increase in performance will require the pressure profile to be further broadened by extending the radius of the ITB. More recent experiments were carried out in 2002 (campaign C5) to move outwards the $q=2$ low shear magnetic shear region by ramping-up the plasma current up to $3.8 \mathrm{MA}\left(q_{95}=2.9\right)$.

Finally, the performance of the two types of JET ITB regimes are compared in figure 2 (right), where the peak neutron rate is plotted versus the additional power for weak and negative central magnetic shear configuration. Heating power in the range of $16 \mathrm{MW}$ is required to generate a wide transport barrier and high fusion yield in the negative central magnetic shear configuration, instead of $20 \mathrm{MW}$ in the weak magnetic shear cases [8]. 

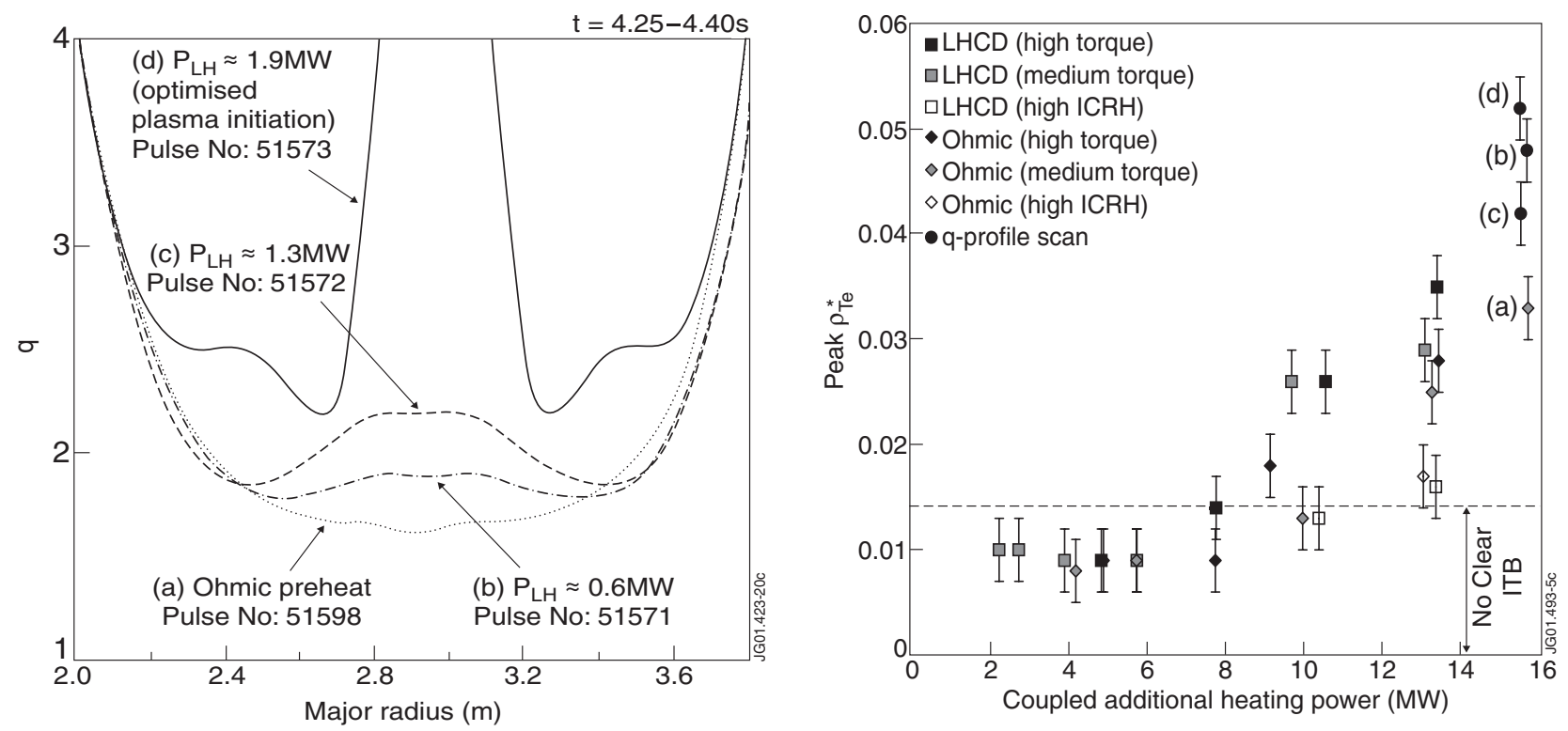

Figure 1. (Left) Various target $q$-profiles prepared with varying LHCD prelude (EFIT equilibrium reconstruction constrained by MSE data). (Right) Maximum value of $\rho_{\mathrm{Te}}^{*}$ versus coupled power for various $q$-profile and heating scenarios $(B=2.6 \mathrm{~T})$; Annotations (a)-(d) refer to the $q$-profile scan shown in the left panel [8]. In the 'high ICRH' cases the ratio of the ICRH power to the total power is above 50\%. This ratio is below $30 \%$ in the dominant NBI cases referred to as 'high' or 'medium' torque and where at a given power level the injected torque has been varied by changing the neutral beam configuration from normal ('medium torque') to tangential ('high torque') beam injection.

\section{Development of ITB plasmas with steady-state potential}

An approach towards stationary operation has been investigated by maintaining the LH power during the highpower ELMy $\mathrm{H}$-mode phase in order to slow down the current profile evolution [9-11,22-24]. Figure 3 shows one of the longest pulses with LH power combining an ELMy H-mode edge (high-frequency type-III ELMs) and core transport barrier sustained with a loop voltage, $V_{\mathrm{s}}$, approaching zero $\left(I_{\mathrm{p}}=2 \mathrm{MA}, q_{95}=5.5, H_{\mathrm{ITER}-89 \mathrm{P}} \sim 2, H_{\mathrm{IPB} 98(\mathrm{y}, 2)} \sim 1\right.$, $\beta_{\mathrm{p}}=1.1, \beta_{\mathrm{N}}=1.7$ at $\left.B_{0}=3.45 \mathrm{~T}\right)$. Successful coupling of the $\mathrm{LH}$ waves during the $\mathrm{H}$-mode phase has been obtained by increasing the density at the antenna mouth by locally injecting $\mathrm{CD}_{4}$ gas $[24,25]$. The line-averaged electron density measured close to the edge transport barrier with the type-III ELM activity maintains a constant value during the high-power phase at typically $1.5 \times 10^{19} \mathrm{~m}^{-3}$. The particle sources at the edge are determined by the plasma recycling at the wall and the injection of $\mathrm{CD}_{4}$ gas. In this discharge, the electron ITB is maintained during $11 \mathrm{~s}$ from the $\mathrm{LH}$ preheat phase up to the pre-programmed end of the power waveforms. This is the longest discharge during which an ITB has been sustained on JET and this duration corresponds approximately to 37 energy confinement times, $\tau_{\mathrm{E}}$. These ITB durations become comparable to the volume-averaged resistive current diffusion time evaluated with the local neo-classical conductivity profile. The ITB also observed in the ion temperature, electron density and toroidal rotation profiles is sustained during the high-power phase $\left(\sim 8 \mathrm{~s}, \sim 27 \tau_{\mathrm{E}}\right)$ starting at $t=4.2 \mathrm{~s}$. The core electron density rises up to $n_{\mathrm{eo}}=6.0 \times 10^{19} \mathrm{~m}^{-3}$ mainly due to the fuelling of core NBI and the line-averaged density normalized to the Greenwald density is 0.55 . The duration of this type of discharge is close to the JET technical operational limits fixed by the maximum duration of application of the full NBI power and the high toroidal field operation.

The target $q$-profile is non-monotonic with $q_{\text {min }} \sim 3$ at $r / a \sim 0.5$, as inferred from the EFIT code constrained by MSE measurements or FIR polarimetry data. In the high-power phase, the $q$-profiles maintain a non-monotonic shape with $q_{\text {min }}$ maintained above 2 at mid-radius. The FIR polarimetry data indicate that $q_{\min }$ is at mid-radius and slowly decreases from 3 down to approximately 2.5 just at the end of the highpower phase. The internal inductance, $l_{i}$, maintains a low value $(\sim 0.8)$ up to the end of the high-power phase and weakly evolves by less than $10 \%$. The slow evolution of the $q$-profile, in particular the location and value of $q_{\mathrm{min}}$, allows to maintain the ITBs inside the mid-plasma radius, i.e. in the weak or negative magnetic shear region, without reaching the disruptive ideal $n=1$ kink limit. The $q$-profile evolution is slowed down due to the high fraction of non-inductive current reaching up to $90 \%$ of the total current as calculated by TRANSP or CRONOS $[26,27]$. The non-inductive current fraction is fairly constant with time: when the electron density is increased the LH and NB non-inductive currents are both reduced, but this effect is partly compensated by the rise of the bootstrap current. The self-consistent CRONOS simulations of the various noninductive currents with two-dimensional equilibria indicate that the off-axis bootstrap current rises up to 1.0 MA, the NBCD varies between 0.2 and $0.6 \mathrm{MA}$, whereas the LHCD deduced from ray-tracing coupled to two-dimensional FokkerPlanck modelling is in the range of $0.4-0.8 \mathrm{MA}$ (figure 3 (right)) [10]. The LH ray-tracing simulations during the highpower phase show that the LH power is absorbed in a broad off-axis region thanks to the strong electron Landau damping with high electron temperature.

The possibility to sustain the ITB characteristics offers the opportunity of quantitatively studying the transport of 

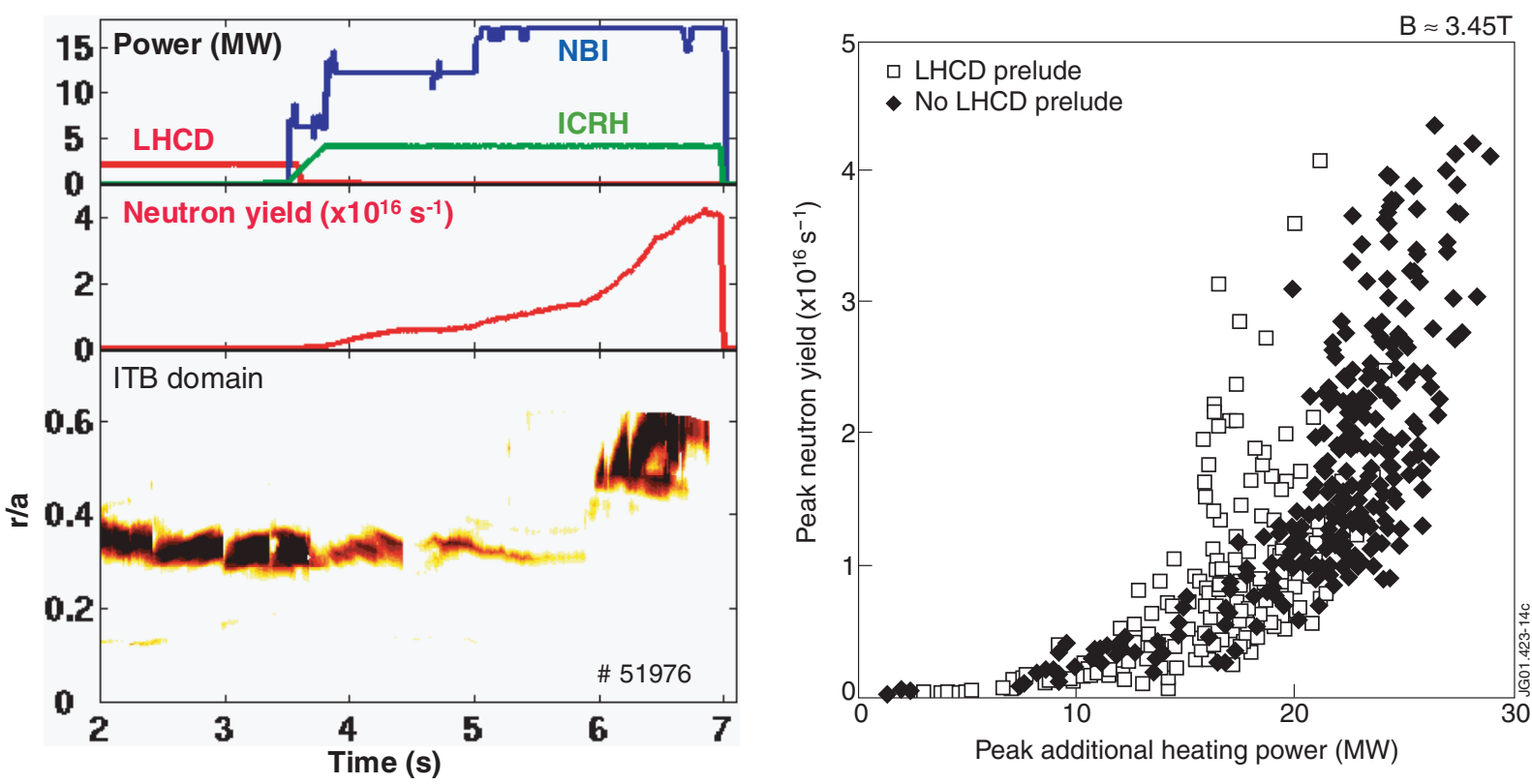

Figure 2. (Left) Time evolution of applied powers, neutron yield and ITB radius, as defined in [7], of a high fusion performance negative central magnetic shear discharge (\#51976). (Right) Peak neutron yield versus additional heating power for the weak and negative magnetic shear magnetic configuration [8] (data from experiments with the JET MKII Gas-Box divertor configuration with the septum part).
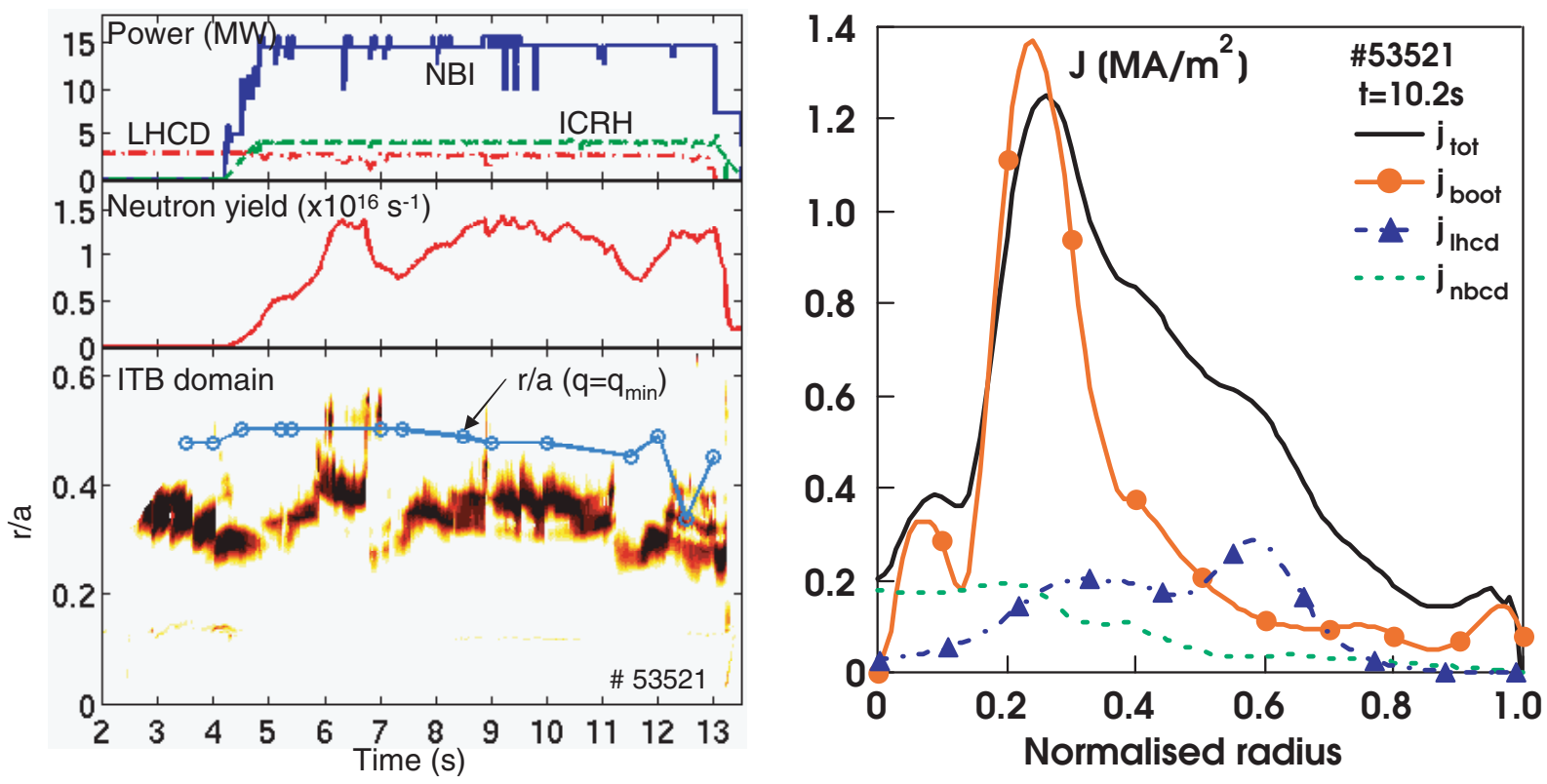

Figure 3. (Left) Time evolution of applied powers, neutron yield, ITB radius (as defined in [7]) and $q_{\text {min }}$ normalized radius of a highly non-inductive current discharge (\#53521). (Right) Current density profiles of bootstrap, LH, neutral beam and total deduced from current diffusion simulation performed with the CRONOS code [10].

particles and impurities. Such studies require that the regime is maintained on a time duration of at least of the order of the particle confinement time (several $\tau_{\mathrm{E}}$ ). These studies have shown that the core impurity transport follows the trends predicted by the neo-classical theory: high $Z$-impurity accumulation with strong peaking of the density profile competing with the screening effect expected from a high ion temperature gradient $[28,29]$. In the experiment shown in figure 3, the screening effect is either too localized and/or not strong enough to prevent the central accumulation of the metallic impurity. The high- $Z$ impurity accumulation (central nickel density rising up to $4 \times 10^{17} \mathrm{~m}^{-3}$ ) leads to a core radiative collapse $(t=11.1 \mathrm{~s})$ in which energy and density are expelled. The radiative power density in the plasma core $(r / a<0.3)$ increases up to $140 \mathrm{~kW} \mathrm{~m}^{-3}$ (at $t=11.1 \mathrm{~s}$ ), which is indeed approaching the conducted electron power density. Control of the impurity content will be investigated either by triggering core MHD activity or by controlling the density and temperature profiles (cf section 4).

One of the surprising results of this experiment is that the ITB is re-formed immediately after the core MHD $(t \approx 6.7 \mathrm{~s})$ 


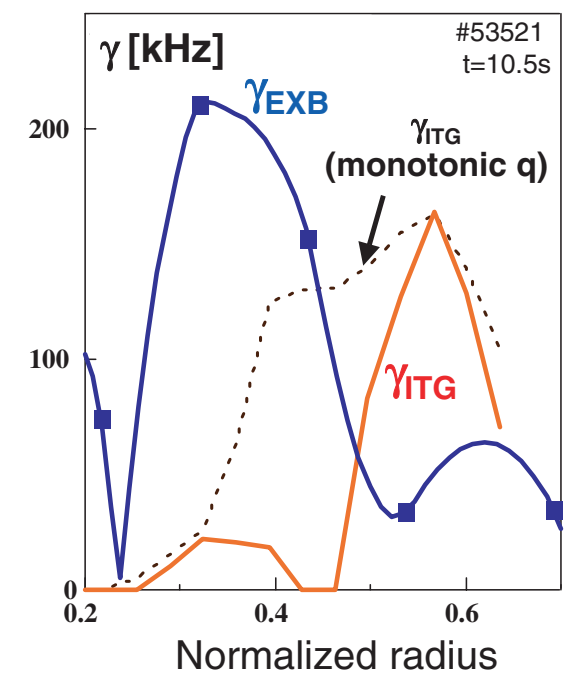

Figure 4. Linear growth rates, $\gamma$, obtained by the gyrokinetic code KINEZERO for the low wavenumbers such as $k_{\theta} \rho_{\mathrm{i}} \leqslant 1$. The full line corresponds to the simulation performed with the measured non-monotonic $q$-profile (pulse \#53521 at $t=10.5 \mathrm{~s}$ ). The dashed line corresponds to a monotonic $q$-profile case with positive magnetic shear. The radial profile of the $E \times B$ shearing rate, $\gamma_{E \times B}$ is shown for comparison.

or radiative $(t \approx 11.1 \mathrm{~s})$ collapses. These collapses affect on a fast time scale the pressure, toroidal rotation and radial electric field profiles, but the $q$-profile, evolving on a longer time scale, keeps a non-monotonic shape. Simulations shown in figure 4 , performed with the electrostatic linear gyrokinetic code, KINEZERO [30], indicate that the ion instabilities driven by the ITG and the trapped electron modes are suppressed in the region of negative magnetic shear [10,31-33]. Therefore, the direct reduction or stabilization of the turbulence through the $q$-profile is invoked to explain the rapid recovery of the ITB to various collapses as long as these perturbations affect only weakly the low or negative magnetic shear region.

One of the challenges for the viability of steadystate plasmas consists in successfully combining the $\mathrm{H}$-mode confinement with the ITB characteristics. Highperformance ITB discharges occur typically at power levels well above the $\mathrm{L}$ - to $\mathrm{H}$-mode transition and even above the type-III to type-I ELM transition. When a transition to largeamplitude ELMs occurs, the ITB is either lost with a backtransition to a lower confinement state or the whole plasma becomes unstable leading to a major disruption. Although the total applied power is at least a factor two above the power threshold to trigger large-amplitude ELMs, the ELMs activity maintains a mild type-III behaviour in these highly noninductive current discharges even without injecting radiative gases for edge cooling. The mitigation of the activity of the ELMs has been interpreted by analysing the role played by the broad $q$-profile with low $l_{\mathrm{i}}$ values $\left(l_{\mathrm{i}} \sim 0.7-0.8\right)$ on the threshold conditions from type-III to type-I ELM $[34,35]$. A larger current fraction at the plasma 'edge' in the ITB discharges compared to the similar standard $\mathrm{H}$-mode ones could prevent a transition to type-I ELM by changing the edge MHD stability conditions. To characterize the edge current fraction, the internal inductance $\left(l_{\mathrm{i}}\right)$, the edge magnetic shear $\left(\mathrm{sh}_{95}\right)$ and the polarimetry measurements (in particular the line of sight close the edge, $R \sim 3.75 \mathrm{~m}$ ) have been simultaneously used. It was checked if the ITB discharges with a high fraction of non-inductive current have larger edge current compared to the standard $\mathrm{H}$-mode regime in agreement with the polarimetry measurements together with the lower values of $l_{\mathrm{i}}$ and $\mathrm{sh}_{95}[34,35]$. The broad current profile with a larger edge current fraction (compared to the H-mode regime) is sustained throughout the high-power phase since a large fraction of off-axis non-inductive current is continuously driven in these long-duration ITB discharges.

This edge issue has been further investigated in the 2002 experimental campaign in similar operating conditions but at high triangularity, $\delta \sim 0.45$, where the ELM activity is expected to be even stronger. So far, JET ITB experiments were mainly performed at reduced values of triangularity $(\delta \sim 0.2)$. The recent modification of the divertor geometry (the septum part of the MKII Gas Box has been removed) has allowed ITB operation with plasma equilibria at triangularity closer to the values envisaged for ITER advanced mode of operation. The ELM behaviour has been changed (at fixed deuterium gas injection rate) by varying the plasma current once the negative central magnetic shear configuration was established. In the experiment shown in figure 5, the full power is applied on a 2 MA current plateau where type-I ELM activity is triggered. Then, the current is raised by $0.3 \mathrm{MA}$, inducing an increase of the edge current ('skin effect') that destabilizes the $n=1$ peeling modes. As a consequence, the type-I ELM activity is suppressed, confirming the role played by the edge current density on the ELM behaviour.

\section{Real-time feedback control of the ITB plasmas for steady-state operation}

New real-time measurements and control algorithms have been developed and implemented for controlling the ITB dynamics and the current density profile: confinement parameters, temperatures, particle and $q$-profiles are now available in real-time.

A double-loop feedback control with combined ICRH and NBI has been first applied in the non-inductive current drive operational regime, as described in section 3 [11]. The NBI power controls the total neutron yield, $R_{\mathrm{NT}}$, at a given reference and consequently the total bootstrap current fraction through the control of the core plasma pressure. In addition, the ICRH is simultaneously used as an actuator to control the normalized electron temperature gradient in the barrier region, i.e. the maximum value of $\rho_{\mathrm{Te}}^{*}=\rho_{\mathrm{s}} / L_{\mathrm{Te}}$ (figure 6 (left)). For this purpose, the local ITB criterion [7] characterizing the spacetime evolution of the ITBs has been calculated in the real-time system from the ECE radiometer data. The LHCD power is maintained in a pre-programmed way to provide the correct $q$-profile ensuring core confinement and MHD stability. In the example shown in figure 6 (left), the values of the maximum $\rho_{\mathrm{Te}}^{*}$ and the neutron rate are maintained constant, respectively, at 0.025 and $0.9 \times 10^{16}$ neutrons $\mathrm{s}^{-1}$. The active control of the temperature gradient provides an indirect way of acting on the current density profile through the bootstrap current ( $\left.I_{\text {Boot }} \sim 0.9 \mathrm{MA}, I_{\mathrm{p}}=1.8 \mathrm{MA}\right)$. The control of the ITB is applied during $7.5 \mathrm{~s}$ until the end of the pre-programmed power 

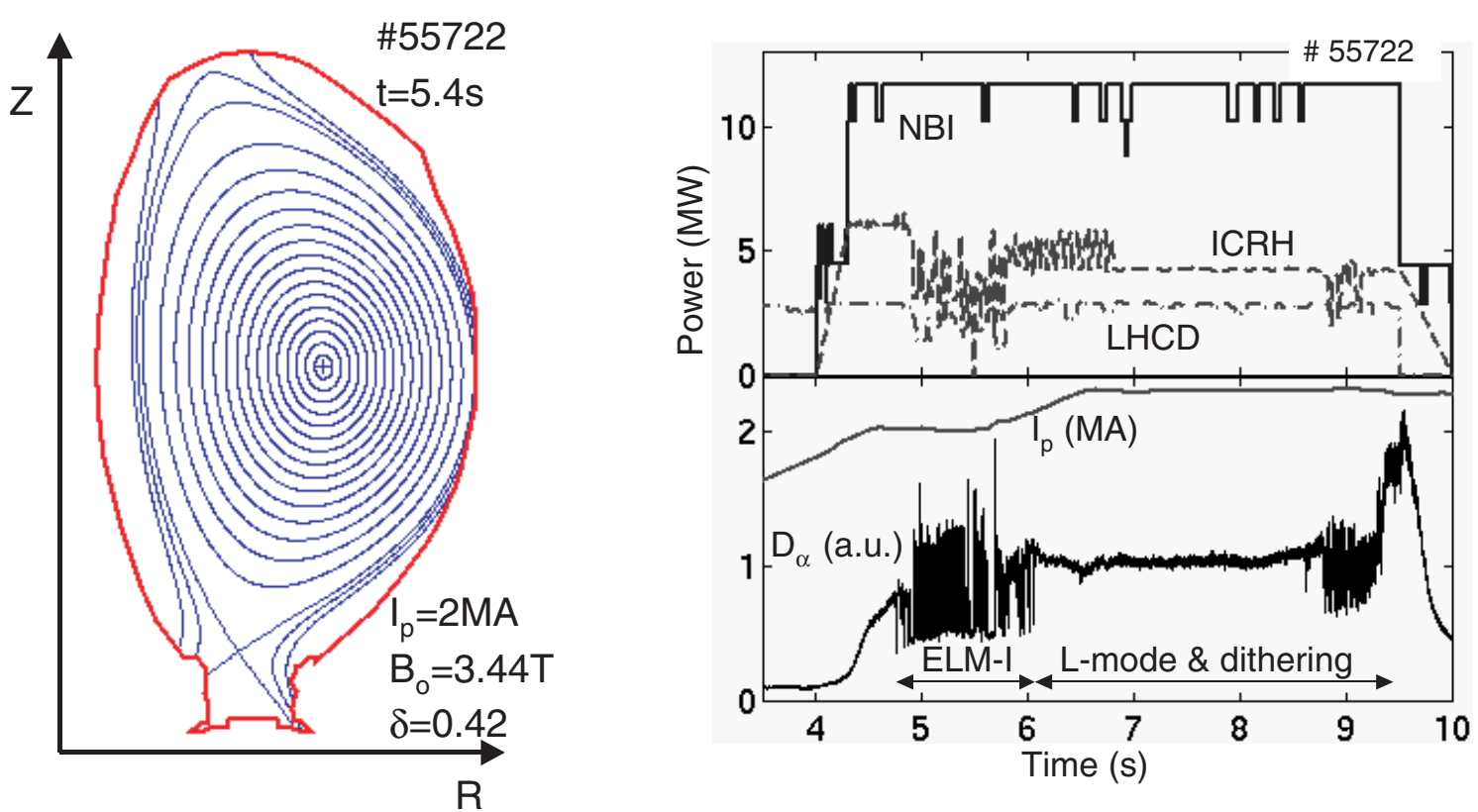

Figure 5. Magnetic equilibria ( $t=5.4 \mathrm{~s}$ ) and time evolution of coupled powers (NBI, ICRH and LHCD), $I_{\mathrm{p}}$ and $D_{\alpha}$-emission in the ELM control experiment at high $\delta$ (\#55722). The ITB is sustained up to $t \sim 5 \mathrm{~s}$ and disappears as a consequence of the type-I ELM activity. Experiment carried out with the MKII Gas Box divertor without the septum part.
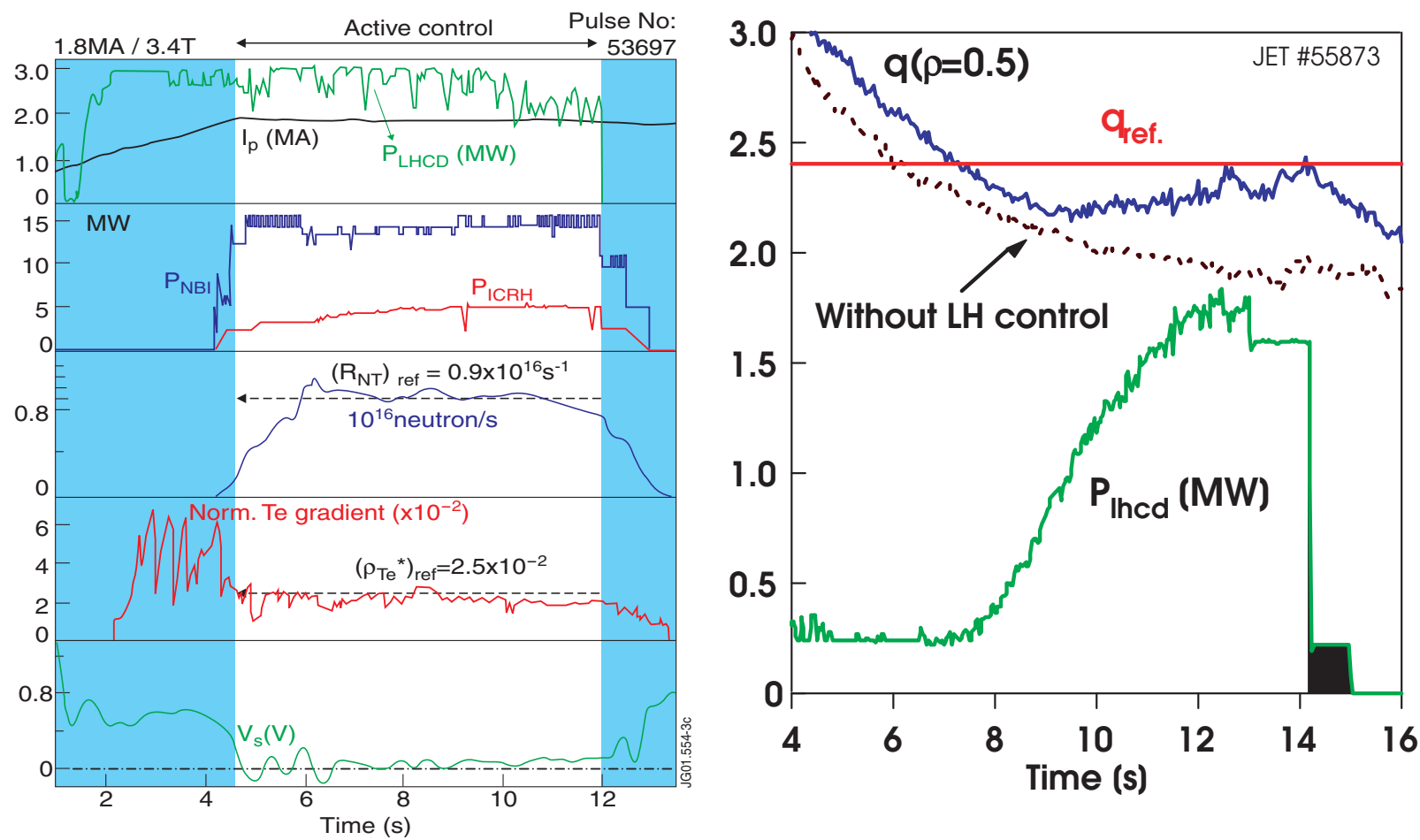

Figure 6. (Left) Time evolution of the main parameters of a discharge with combined $\rho_{\mathrm{T}}^{*}$ and $R_{\mathrm{NT}}$ feedback control using ICRH and NBI powers (\#53697) [11]. (Right) Real-time $q$-profile control carried out in an extended LHCD prelude phase: time evolution of LHCD power, measured and reference $q$-values at mid-radius (\#55873). A discharge (\#55872) without LHCD feedback control is presented for comparison (- - -) [36].

waveform envelope within which the NBI and ICRH powers are allowed to vary. During the control phase, the plasma parameters of the discharge are fairly stationary with mild and continuous ELM activity. Thanks to the real-time control of the ITB characteristics, the improved confinement state is maintained in a more reproducible and stationary manner, e.g. avoiding the occurrence of the core collapses (MHD or radiative collapses).

Recent efforts have been made in order to develop an algorithm that provides a measurement of the $q$-profile in realtime and allows feedback control $[36,37]$. The algorithm uses as inputs the signals of the magnetic and interfero-polarimeter 
diagnostics. The approach described is based on decoupled loops controlling the core pressure and temperature gradient at two radii with devoted actuators. A 'model-based' profile control scheme is now followed, in which more information on the spatial structure of the system is taken into account by retaining its distributed nature, and considering the non-local interaction between various quantities through a diffusion-like operator [38]. To validate this 'model-based' technique a direct control of the safety factor profile has been tried using LHCD as the only actuator. The experiment was performed during an extended LHCD prelude phase at low density and beta. The plasma current was fixed at 1.5 MA, in order to be close to a purely non-inductive current drive regime with the available LH power and thus have a larger flexibility for obtaining weak shear $q$-profiles. The feedback control was performed on five points of the $q$-profile located at fixed normalized radii $(r / a=0.2,0.4,0.5,0.6,0.8)$ and the reference $q$-profile is reached within $4 \mathrm{~s}$ (figure 6 (right)). To reach the preset reference $q$-profile the controller minimizes in the leastsquares sense the difference between the five target $q$-values and the real-time measurements. Modelling of the current diffusion evolution using the CRONOS code [26] confirms that a stationary state with a flat toroidal electric field profile was reached (the resistive time is around $4 \mathrm{~s}$ in these experiments). The simulations also indicate that 0.9 MA of non-inductive current is driven by the $\mathrm{LH}$ waves absorbed at mid-radius.

The successful experiment reported here should be considered as a 'proof of principle'. In the near future, this general 'model-based' approach will be implemented in view of controlling high- $\beta$, high-bootstrap fraction, ITB discharges where pressure and current density profiles are strongly nonlinearly coupled.

\section{Conclusion and prospects}

Using off-axis LH current drive, the previously developed Optimized Shear regime [3] with low or weakly negative magnetic shear in the plasma centre has been extended up to noticeably negative central magnetic shear configurations [8]. To conclude, the performances of these ITB plasmas quantified by the product of the $H_{\text {ITER } 89 \mathrm{P}} \times \beta_{\mathrm{N}}$ have been plotted in figure 7 versus the total injected energy, i.e. applied power times the discharge duration. This graph summarizes the results achieved with ITB plasmas and negative central magnetic shear configuration over the JET experimental period extending from June 2000 up to June 2002 (experimental campaigns $\mathrm{C} 1-\mathrm{C} 5)$ :

- High-performances with wide ITB $(r / a \sim 0.6)$ triggered when $q_{\min }$ reaches a low-order rational surface (two) have been achieved transiently (during $\sim 2 \tau_{\mathrm{E}}$ ) at $H_{\text {ITER-9P }} \sim 3.3, H_{\mathrm{IPB} 98(\mathrm{y}, 2)} \sim 1.9, \quad \beta_{\mathrm{N}}=2.4$ $\left(I_{\mathrm{p}}=2.5 \mathrm{MA}, B_{0}=3.45 \mathrm{~T}, q_{95}=4.5\right)$ with $40 \%$ of bootstrap current and $55 \%$ of non-inductive current [8].

- Quasi-stationary regimes with narrower ITB $(r / a \sim 0.4)$ and mild type-III ELM activity have been sustained on a resistive time scale $\left(\sim 37 \tau_{\mathrm{E}}\right)$ at $H_{\mathrm{ITER}-89 \mathrm{P}} \sim 2$, $H_{\mathrm{IPB} 98(\mathrm{y}, 2)} \sim 1, \beta_{\mathrm{N}}=1.7\left(I_{\mathrm{p}}=2 \mathrm{MA}, B_{0}=3.45 \mathrm{~T}\right.$, $q_{95}=5.5$ ) with $50 \%$ of bootstrap (1 MA) and up to $100 \%$ of non-inductive current $[9,10]$.
In addition, the feasibility to control in real-time the ITB characteristics (e.g. the electron temperature gradient at the ITB location) with feedback loops has been demonstrated in the highly non-inductive current drive regime. The sustainability and the real-time control of the ITBs in full current drive operation with a significant fraction of bootstrap current represent a major milestone towards the definition and viability of the steady-state tokamak operation.

Nevertheless, despite these favourable results for the advanced mode of operation, plasma performances quantified, for instance, in terms of the normalized pressure (e.g. $\beta_{\mathrm{N}}$ ) of the JET non-inductive quasi-steady-state regime should be increased further. Figure 8 summarizes the results obtained

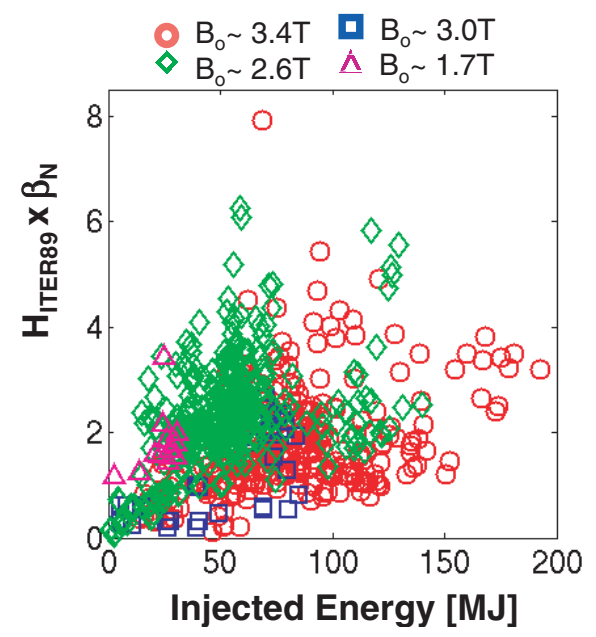

Figure 7. The maximum value of $H_{\text {ITER89-P }} \times \beta_{\mathrm{N}}$ plotted versus the total injected energy at various toroidal fields $\left(B_{0} \sim 3.4 \mathrm{~T}\right.$, $\left.B_{0} \sim 3.0 \mathrm{~T}, B_{0} \sim 2.6 \mathrm{~T}, B_{0} \sim 1.7 \mathrm{~T}\right)$. The total injected energy is the sum of the injected LHCD, NB and ICRH energies. Each point corresponds to one discharge. The data correspond to ITB experiments carried out during the JET-EFDA experimental campaigns (C1-C5) from June 2000 up to June 2002.

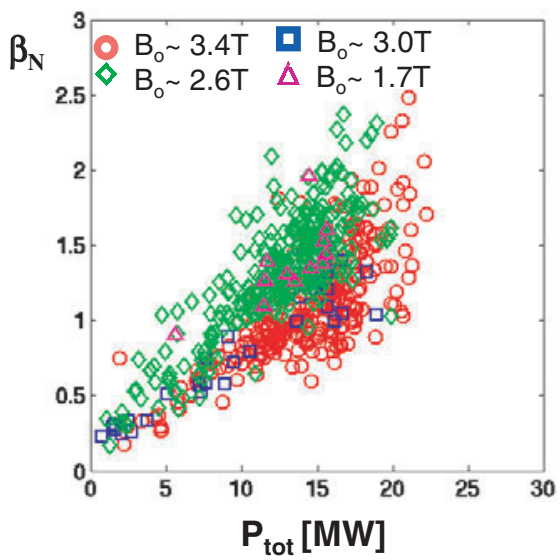

Figure 8. Normalized (thermal and non-thermal) pressure, $\beta_{\mathrm{N}}$, versus the total injected power, $P_{\text {tot }}$, at various toroidal fields $\left(B_{0} \sim 3.4 \mathrm{~T}, B_{0} \sim 3.0 \mathrm{~T}, B_{0} \sim 2.6 \mathrm{~T}, B_{0} \sim 1.7 \mathrm{~T}\right)$. The total injected power is the sum of the LHCD, NBI and ICRH powers. Each point corresponds to one discharge. The $\beta_{\mathrm{N}}$ and $P_{\text {tot }}$ values have been obtained at the time of the maximum value of the neutron yield. The data correspond to ITB experiments carried out during the JET-EFDA experimental campaigns (C1-C5) from June 2000 up to June 2002. 
so far in the JET ITB regime where $\beta_{\mathrm{N}}$ is plotted versus the total applied power at various toroidal fields. Thanks to the enhanced capabilities of the improved heating systems (NBI and ICRH) for future experimental campaigns, we are aiming at $\beta_{\mathrm{N}} \sim 3$ for quasi-steady state ITB plasmas [39]. One obvious route to pursue to reach this goal in quasi-steady state consists in extending the radius of the ITB for embracing a larger plasma volume with reduced transport. We have shown that a wide ITB with a steep gradient could be formed when the low magnetic shear region is moved outwards closer to the low-order rational $q$-surface (e.g. $q=2$ ). A major challenge remains to sustain and control towards stable steadystate conditions the characteristics of the wide ITBs together with the required $q$-profile for confinement and stability. The real-time measurements of the kinetic and magnetic profiles together with the 'model-based' feedback control algorithms will be extensively used in future experimental campaigns in view of further increasing the plasmas fusion performance in a quasi-steady-state manner. In addition, effort will be made to develop a non-inductive mode of operation at high triangularity $(\delta \sim 0.5)$ as required in the present ITER design.

\section{Acknowledgments}

This work has been performed under the European Fusion Development Agreement (EFDA). It is a pleasure to acknowledge the contributors to the EFDA-JET work programme, in particular those involved in the Task Force S2 and in the Task Force $\mathrm{H}$, as well as the UKAEA, operator of the EFDA-JET facility, and the EFDA Close Support Unit in Culham.

\section{References}

[1] Taylor T.S. 1997 Plasma Phys. Control. Fusion 39 B47

[2] Litaudon X. 1998 Plasma Phys. Control. Fusion 40 A251

[3] Gormezano C. 1999 Plasma Phys. Control. Fusion 41 B367

[4] Wolf R.C. 2003 Plasma Phys. Control. Fusion 45 R1

[5] Special issue on Advanced tokamak research in EFDA-JET during the 2000-2001 experimental campaigns 2002 Plasma Phys. Control. Fusion 441031

[6] Bécoulet A. et al 2001 Plasma Phys. Control. Fusion 43 A395

[7] Tresset G. et al 2002 Nucl. Fusion 42520

[8] Challis C.D. et al 2002 Plasma Phys. Control. Fusion 441031

[9] Crisanti F. et al 2002 Phys. Rev. Lett. 88 145004-1

[10] Litaudon X. et al 2002 Plasma Phys. Control. Fusion 441057

[11] Mazon D. et al 2002 Plasma Phys. Control. Fusion 44 1087
[12] JET Team (prepared by Gormezano) 1999 Nucl. Fusion 391875

[13] Söldner F.X. et al 1999 Nucl. Fusion 39407

[14] Challis C.D. et al 2001 Plasma Phys. Control. Fusion 43861

[15] Hawkes N. et al 2001 Phys. Rev. Lett. 87115001

[16] Huysmans G.T.A. et al 2001 Phys. Rev. Lett. 87245002

[17] Tala T.J.J. et al 2002 Plasma Phys. Control. Fusion 441181

[18] Joffrin E. et al 2002 Nucl. Fusion 42235

[19] Joffrin E. et al 2002 Plasma Phys. Control. Fusion 441739

[20] Garbet X. et al 2001 Phys. Plasmas 82793

[21] Garbet X. et al 2002 Micro-stability and transport modelling of internal transport barrier on JET Proc. 19th Int. Conf. (Lyon, 2002) (Vienna: IAEA)

[22] Mailloux J. et al 2002 Phys. Plasmas 92156

[23] Castaldo C. et al 2002 Phys. Plasmas 93205

[24] Tuccillo A. et al 2001 RadioFrequency power in plasmas Proc. 14th Top. Conf. (Oxnard, CA, 2001) vol 595, ed T.K. Mau and J. de Grassie (New York: American Institute of Physics) p 209

[25] Pericoli V. et al 2003 Study and optimization of lower hybrid waves coupling in advanced scenario plasmas in JET Plasma Phys. Control. Fusion submitted

[26] Basiuk V. et al 2003 Simulations of steady-state scenarios for Tore Supra using the CRONOS code Nucl. Fusion at press

[27] Hoang G.T. et al 2002 Confrontation of heat transport models against Tore Supra and JET high performance discharges Proc 19th Int. Conf. (Lyon, 2002) (Vienna: IAEA)

[28] Dux R. et al 2001 Proc. 28th Eur. Conf. (Madeira) vol 25A (EPS) p 505

[29] Dux R. et al 2003 J. Nucl. Mater. 313-316 1150

[30] Bourdelle C., Garbet X., Hoang G.T., Ongena J. and Budny R.V. 2002 Nucl. Fusion 42892

[31] Eriksson L.-G. et al 2002 Phys. Rev. Lett. 88 145001-1

[32] Fourment C., Hoang G.T., Eriksson L.-G, Garbet X., Litaudon X. and Tresset G. 2003 Plasma Phys. Control. Fusion 45233

[33] Esposito B. et al 2002 Correlation between magnetic shear and $E \times B$ shear flow on JET ITBs Proc. 29th Eur. Conf. on Plasma Physics and Controlled Fusion (Montreux, 17-21 June 2002) (EPS) vol 26B (http://crppwww.epfl.ch/eps2002/)

Esposito B. et al 2003 Plasma Phys. Control. Fusion 45933

[34] Bécoulet M. et al 2002 Plasma Phys. Control. Fusion 44 A103

[35] Sarazin Y. et al 2002 Plasma Phys. Control. Fusion 442445

[36] Mazon D. et al 2002 Real-time control of pressure and current profile in JET Proc. 29th Eur. Conf. on Plasma Physics and Controlled Fusion (Montreux, 17-21 June 2002) (EPS) vol 26B (http://crppwww.epfl.ch/eps2002/)

Mazon D. et al 2003 Plasma Phys. Control. Fusion 45 L47

[37] Zabeo L. et al 2002 Plasma Phys. Control. Fusion 442483

[38] Moreau D. et al 2003 Real time control of internal transport barriers in JET Proc. 3rd IAEA TCM on Steady State Operation of Magnetic Fusion Devices (May 2002) Nucl. Fusion submitted

[39] Pamela J. et al 2002 Nucl. Fusion 421014 